\title{
Traqueostomía percutánea con técnica Ciaglia Blue Rhino. Experiencia en Hospital Clínico FUSAT de Rancagua
}

\author{
Percutaneous tracheostomy Ciaglia Blue Rhino technique. \\ Revision 2 years of experience
}

\author{
Ricardo Quitral C', Carolina Caulier F'1, Pilar Rubio V², Carlos Aguayo Z³.
}

\begin{abstract}
RESUMEN
Introducción: La traqueostomía descrita ya hacia el 1500 AC, en las últimas décadas ha evolucionado al desarrollo de sistemas de abordaje mínimamente invasivos o percutáneos, de los cuales la técnica de Ciaglia Blue Rhino (dilatador único), es la más utilizada en la actualidad.

objetivo: Se realizó un estudio retrospectivo descriptivo de las traqueostomías realizadas en el Hospital Clínico FUSAT de Rancagua, entre los años 2006 y 2012, con el fin de evaluar ventajas, desventajas e incidencia de complicaciones de la técnica abierta versus la técnica percutánea en nuestras manos.

Material y método: Se incluyeron 64 traqueostomías consecutivas de las cuales 42 fueron percutáneas con técnica de Ciaglia Blue Rhino y el resto con técnica abierta clásica.

Resultados: Al analizar los resultados encontramos ventajas de la técnica percutánea como un importante acortamiento del tiempo quirúrgico (de 45,27 minutos en la técnica clásica a 15,28 minutos promedio en la técnica percutánea), una menor incidencia de complicaciones (14\% en procedimiento percutáneo versus $27 \%$ en procedimiento abierto) y una curva de aprendizaje relativamente rápida que permitió que la indicación para el procedimiento percutáneo pasara desde $68 \%$ en la primera mitad de la serie al $92 \%$ en la segunda mitad de ésta.

Conclusión: Creemos que la técnica es segura en manos de otorrinolaringólogos y debería incorporarse como una herramienta más al arsenal quirúrgico de la especialidad.

Palabra clave: Traqueostomía, percutánea, técnica Ciaglia Blue Rhino.
\end{abstract}

\begin{abstract}
Introduction: The tracheostomy described as about 1500 BC, only in recent decades has seen the development of systems for minimally invasive or percutaneous, of which the Ciaglia Blue Rhino technique (single dilator), is most often used today.
\end{abstract}

\footnotetext{
1 Médico Servicio de Otorrinolaringología, Hospital Clínico FUSAT.

2 Médico Servicio de Pediatría, Hospital Clínico FUSAT.

3 Médico Anestesista, Hospital Clínico FUSAT.
}

Recibido el 16 de octubre, 2013. Aceptado el 11 de enero, 2015. 
Aim: We performed a retrospective study of tracheostomies performed at the FUSAT Clinical Hospital from Rancagua, between 2006 and 2012, in order to evaluate advantages, disadvantages and incidence of complications of open surgery versus percutaneous technique in our hands.

Material and methods: Included 64 tracheostomies of which 42 were percutaneous Ciaglia Blue Rhino technique.

Results: In analyzing the results highlights shortening surgical time from $45.27 \mathrm{mi}$ nutes for classical technique to 15.28 minutes for percutaneous (statistically significant) in relation to complications, they showed a lower incidence in the group with percutaneous technique, the learning curve of the procedure is quick, reaching its indication in our series more than $90 \%$ of cases once acquired experience.

Conclusion: We believe that the technique is safe in the hands of Otolaryngologists and should be incorporated as another tool to the arsenal surgical specialty.

Key words: Percutaneous, Tracheostomy, Ciaglia Blue Rhino technique.

\section{INTRODUCCIÓN}

Las primeras referencias a la traqueostomía como intervención quirúrgica pueden encontrarse en escritos de entre el 1700 y el 1100 AC y hacia el siglo II DC, Galeno ya había descrito su técnica para la realización del procedimiento, sin embargo, no es hasta 1909 que Chevalier Jackson estandariza la técnica ${ }^{1}$ y las indicaciones para la operación, demostrando que la identificación cuidadosa de algunos puntos de reparo anatómico reducían drásticamente la tasa de complicaciones asociadas a ésta. Desde esa fecha la técnica quirúrgica ha sufrido solo pequeñas modificaciones, en tanto, se ha ido generando mucho interés por el desarrollo de sistemas de abordaje mínimamente invasivos.

La traqueostomía percutánea (TP) fue descrita inicialmente por Shelden y cols en $1955^{2}$, su técnica consistía en la instrumentación cruenta de la tráquea y estuvo asociada a una elevada tasa de complicaciones lo que limitó su popularización; posteriormente se introdujo diversas modificaciones a la técnica con la finalidad de mejorar sus resultados, sin embargo, la era de la TP moderna, parte con la descripción del método de dilatación desarrollado por Ciaglia y cols en $1985^{3}$; esta técnica, aprovecha las bondades del procedimiento de Seldinger (punción directa vascular mediante una aguja con estilete metálico y cánula plástica, seguida de la colocación de una guía en el interior de la aguja), para facilitar la introducción de los dispositivos de diferente calibre que permitían la dilatación progresiva de la tráquea hasta la colocación de la cánula. En 1998, se introduce una modificación al procedimiento reemplazándose el sistema de dilatadores múltiples por un dilatador único, denominándose a esta nueva técnica de Ciaglia Blue Rhino. Este refinamiento a la técnica original de Ciaglia, incorporó un dispositivo especializado para la realización de una dilatación única, mostrando ser superior al sistema de dilatación múltiple ${ }^{4}$. Si bien en la actualidad existen diversas modalidades de traqueostomía percutánea con tasas de complicación variables, la técnica Ciaglia Blue Rhino es la más difundida a nivel nacional e internacional y la que ha demostrado tener el mejor perfil de seguridad al ser comparada con otras técnicas de TP por dilatación ${ }^{5}$. En definitiva la traqueostomía ha ido evolucionando desde un procedimiento quirúrgico complejo realizado tradicionalmente en pabellón; a una intervención que puede ser ejecutada en la $\mathrm{UCI}$, en la misma cama del enfermo y mediante un abordaje percutáneo, redundando lo anterior en que la TP sea hoy un procedimiento ampliamente utilizado en las unidades de pacientes críticos de países desarrollados 6 .

La inicial incertidumbre sobre la seguridad de la TP en comparación con la traqueostomía abierta (TA), se ha ido despejando en los últimos años, encontrándose hoy abundante literatura que no muestra diferencias significativas en complicaciones entre ambas técnicas ${ }^{8-10}$, existiendo sí en las TP un menor índice de complicaciones infecciosas, mejor cicatrización y acortamiento de tiempos quirúrgicos además de beneficios en relación a oportunidad, costo y posibilidad de realización al lado de la cama del paciente ${ }^{8-10}$. 


\section{OBJETIVO}

Evaluar nuestra experiencia utilizando la técnica percutánea de Ciaglia Blue Rhino, comparando ventajas, desventajas y complicaciones entre dicha técnica y la traqueostomía abierta clásica en nuestras manos.

\section{MATERIAL Y MÉTODO}

Se realiza un estudio retrospectivo, descriptivo, de los pacientes sometidos a traqueostomía en nuestro hospital incluyendo 64 traqueostomías consecutivas de las cuales 42 fueron percutáneas y 22 abiertas, para ello, se obtuvo la información del análisis de protocolos operatorios y fichas clínicas de las traqueostomías realizadas en el Hospital Clínico FUSAT de Rancagua entre el 01/05/2006 y el 28/02/2012.

Se incluyeron traqueostomías abiertas y por técnica percutánea, estas últimas realizadas todas en pabellón, en la cama del enfermo, bajo anestesia general, con técnica de dilatador único (Ciaglia Blue Rhino), utilizando kit marca TRACOE en todos los casos (Figura 1) con infiltración prequirúrgica de anestésico con vasoconstrictor de la zona operatoria y utilización de puntos de fijación de cánula a piel con seda 2-0 (ambas acciones no descritas en la técnica original).

Los datos se analizaron en planilla Excel en las categorías de edad, sexo, técnica, cirujano, tiempo quirúrgico, uso de nasofibroscopio y complicaciones (infección, sangramiento, enfisema subcutáneo, desaturación de oxígeno, falsa vía), realizándose posteriormente un análisis estadístico de los datos.

\section{Descripción de técnica}

- Paciente en posición habitual para TQT con cuello en hiperextensión.

- Reconocimiento por palpación de vía aérea (vital en nuestra opinión para la decisión de si se utiliza o no la técnica percutánea).

- Infiltración con anestésico más vasoconstrictor de la zona de punción.

- Retiro por parte del anestesiólogo del tubo traqueal hasta dejar Cuff inmediatamente bajo las cuerdas vocales.

- Bajo el primer anillo traqueal manteniendo estrictamente la línea media, se realiza punción manteniendo fija la vía aérea entre índice y pulgar de la mano que no punciona, con bránula 14 y jeringa cargada con suero 0 agua destilada, manteniendo aspirativo mientras se avanza en la punción, de tal manera que al ingresar en vía aérea se aspire aire.

- Apenas se aspira aire se angula la dirección de la aguja hacia caudal, mientras se avanza ligeramente para asegurarse de que el lumen de la bránula quede en la tráquea (Figura 2).

- Manteniendo fija la bránula con una mano se retira suavemente el conductor metálico con la jeringa para luego introducir el alambre de Seldinger a través de ella (Figuras 3 y 4 ).

- Posicionado el alambre de Seldinger, y evitando que éste retroceda accidentalmente, se retira la

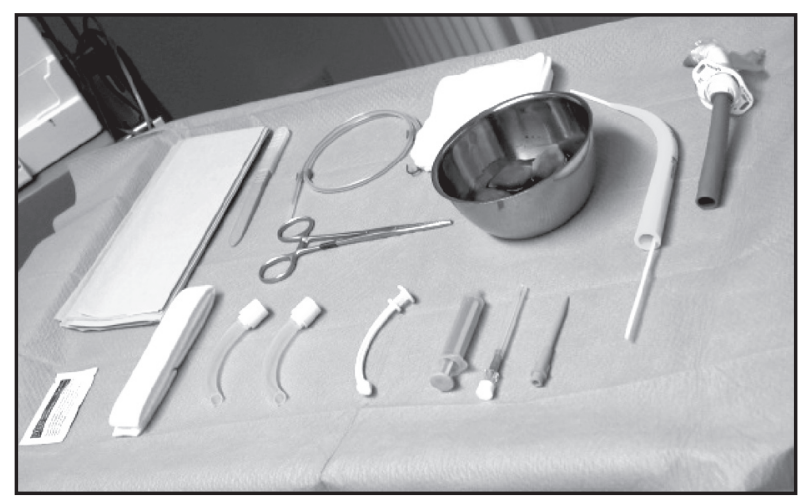

Figura 1. 


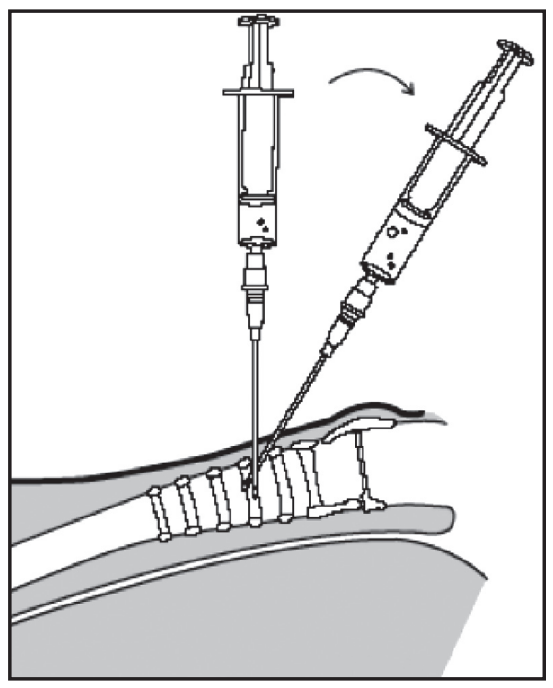

Figura 2.

bránula, manteniendo el alambre en su sitio se amplía la zona de punción cutánea con bisturí en dirección horizontal dejando una incisión de piel de alrededor de $1,5 \mathrm{~cm}$ que permita el paso del dilatador único y posteriormente la cánula, evitando profundizar demasiado el corte para no dañar vasos.

- Se inserta el dilatador corto sobre el alambre, para dilatar el punto de punción, con un movimiento rotatorio suave (el dilatador corto prepara la tráquea para los siguientes pasos) (Figura 4).

- Retiramos el dilatador corto y aplicamos el cateter guía sobre el alambre Seldinger hasta que el tope de seguridad quede sobre el nivel de la piel, previo a esto se esparce el lubricante y moja el dilatador único en suero fisiológico o agua para activar la superficie hidrofílica, después se desliza con facilidad por la activacion del lubricante (Figura 5).

- Finalmente se retira el dilatador, se inserta la cánula precargada en el introductor, después de la inserción de la cánula, se retiran introductor, cateter guía y alambre Seldinger, se introduce la endocánula y conecta al respirador, previo a lo cual, se puede revisar la posición de la cánula con el nasofibroscopio ante cualquier duda (Figura 6).

- Dejamos moltopren en contorno de ostoma y fijamos la cánula a la piel con 4 puntos de seda negra 2-0.

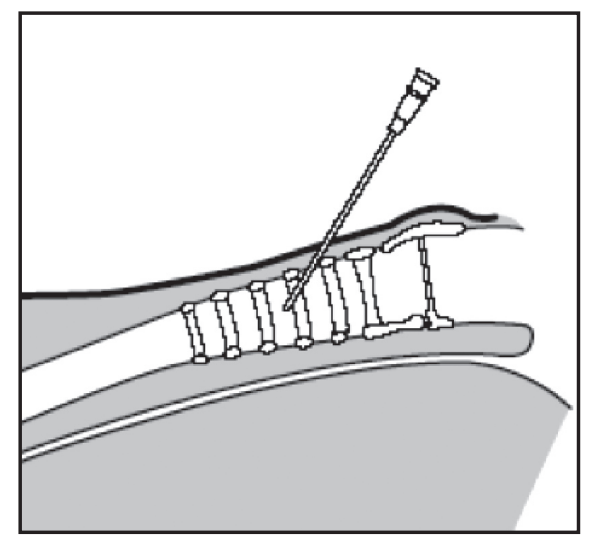

Figura 3

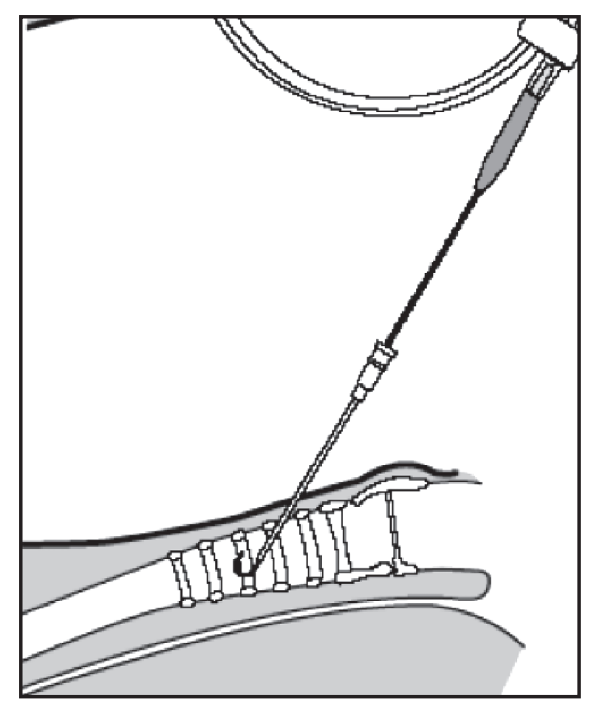

Figura 4.

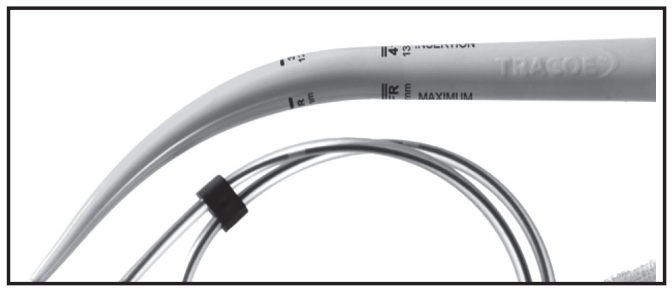

Figura 5.

RESULTADOS

Se realizaron en el periodo estudiado un total de 64 traqueostomías, 22 (34\%) abiertas y 42 (66\%) 


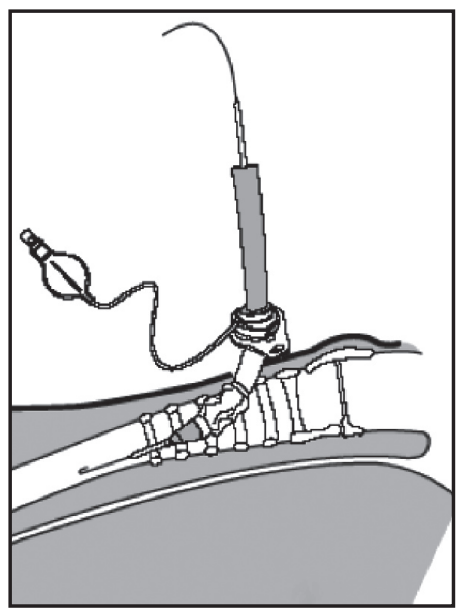

Figura 6.

percutáneas (Figura 7), las indicaciones para el procedimiento fueron ventilación mecánica prolongada en el $93 \%$ de los casos, aspiración en el $1,5 \%$, Ca laríngeo en el $1,5 \%$ y trastorno severo de deglución en el 3\% de los casos; en relación al género de los pacientes, en el $65 \%$ de los casos fue

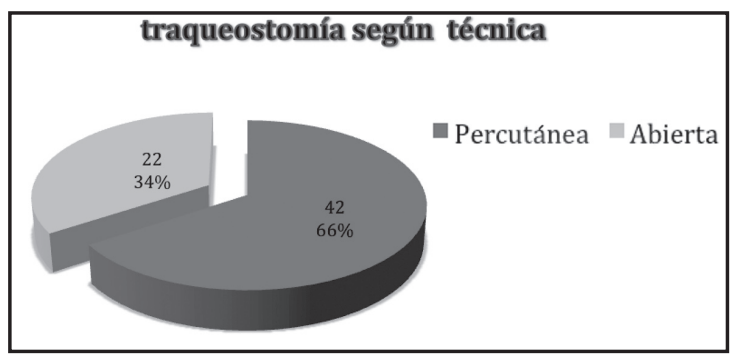

Figura 7.

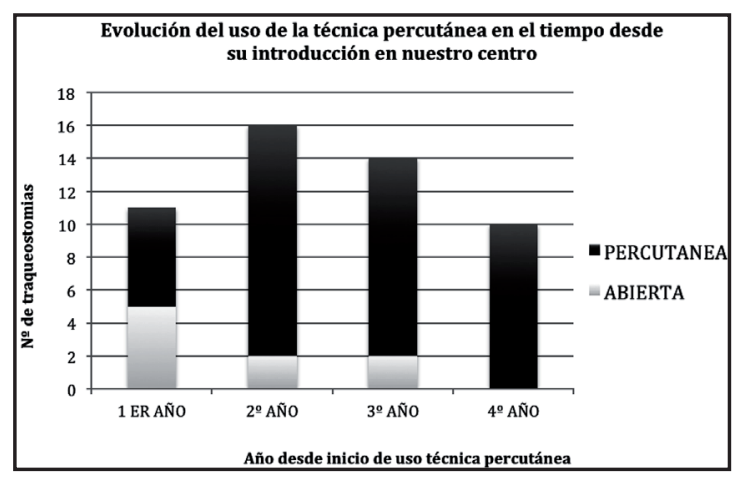

Figura 8. masculino y en el $35 \%$ femenino, la edad promedio para el grupo de traqueostomías percutáneas fue de 64,5 años, con un rango de entre 20 y 86 años, en tanto para las abiertas la edad promedio fue de 67 años con un rango de entre 45 y 94 años; al analizar la proporción de traqueostomías percutáneas versus abiertas por año desde el inicio de la utilización de la técnica percutánea, se observa la rápida adopción de esta técnica por los miembros del equipo, pasando su utilización de cerca del $50 \%$ de los casos en el año de su introducción, al $100 \%$ de los casos en el cuarto año de uso (Figura 8). En relación al tiempo quirúrgico, se observó un menor tiempo quirúrgico en la técnica percutánea (15,28 min promedio) al compararla con la técnica abierta $(45,27$ min promedio) (Figura 9). Complicaciones se presentaron en el $14 \%$ de los pacientes en que se usó técnica percutánea y en el $27 \%$ de los con técnica abierta clásica, respecto a las complicaciones menores, si bien no hubo diferencias estadísticamente significativas al comparar técnica abierta versus percutánea, se observa claramente una menor tendencia a la ocurrencia de ellas con la técnica percutánea (Tabla 1), en nuestra serie no se observaron complicaciones mayores, no hubo defunciones como consecuencia directa del procedimiento con ninguna de las dos técnicas y no tuvimos eventos de decanulación obstrucción. Hubo un episodio de falsa vía (primera mitad de la serie, segundo procedimiento del cirujano, tráquea calcificada). Respecto al uso del fibroscopio, éste fue utilizado en el $11 \%$ de los procedimientos percutáneos, fundamentalmente durante el "periodo de aprendizaje", es decir, en la primera mitad de las realizadas con técnica percutánea.

\section{DISCUSIÓN}

Si bien a nivel nacional ya existen publicaciones con casuísticas importantes (más de 100 casos) que muestran experiencia con traqueostomía percutánea utilizando técnica de Ciaglia Blue Rhino, en las publicaciones aludidas el procedimiento fue realizado por intensivistas o cirujanos y compararon las complicaciones con las descritas en la literatura internacional 
Tabla 1. Incidencia de complicaciones según técnica y su significancia estadística

\begin{tabular}{|c|c|c|c|c|c|}
\hline & \multicolumn{2}{|c|}{ Abiertas (N 22) } & \multicolumn{2}{|c|}{ Percutáneas (N 42) } & \multirow{2}{*}{$\begin{array}{c}\text { Valor } P \text { significativo }<0,05 \\
0,28 \\
\end{array}$} \\
\hline Total de complicaciones & 6 & $27 \%$ & 6 & $14 \%$ & \\
\hline Hemorragia & 2 & $9 \%$ & 1 & $2,4 \%$ & 0,32 \\
\hline Infección & \multicolumn{2}{|c|}{$314 \%$} & 2 & $4,8 \%$ & 0,32 \\
\hline Enfisema Subcutáneo & \multicolumn{2}{|c|}{$14,5 \%$} & \multicolumn{2}{|c|}{$00 \%$} & 0,23 \\
\hline Desaturación 02 bajo 90\% & \multicolumn{2}{|c|}{$00 \%$} & \multicolumn{2}{|c|}{$24,8 \%$} & 0,14 \\
\hline Decanulación obstrucción & 0 & $0 \%$ & 1 & $2,4 \%$ & 0,23 \\
\hline
\end{tabular}

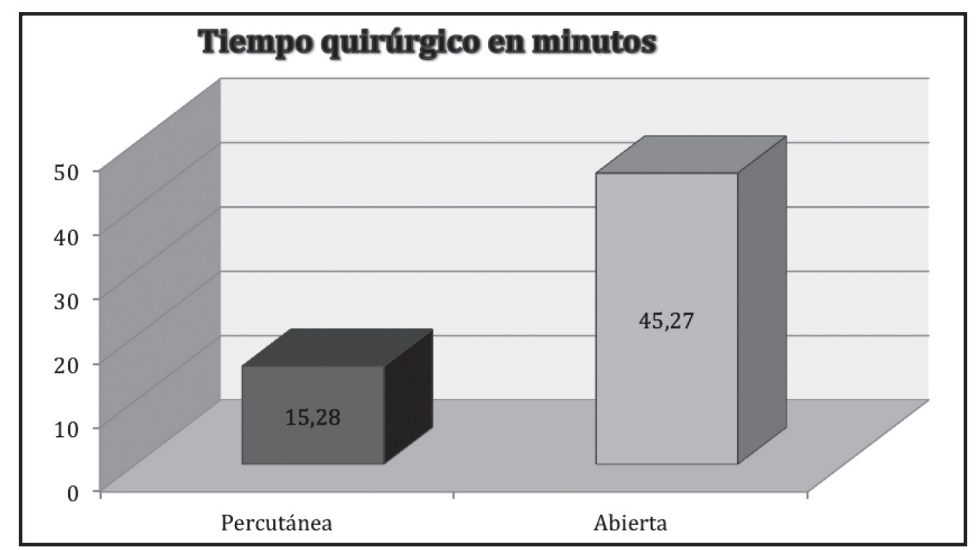

Figura 9.

para el mismo procedimiento sin comparar tiempos ni complicaciones con la técnica abierta clásica ${ }^{10,11}$, a nivel nacional el único estudio comparable con el nuestro es el realizado por Celedón (2007) en el Hospital Clínico de la Universidad de Chile ${ }^{12}$, que muestra un alto índice de complicaciones (40\%), con mínima diferencia en el tiempo quirúrgico, hecho posiblemente explicable por la utilización de KIT de dilatadores múltiples.

En nuestra experiencia el procedimiento ha mostrado un notable acortamiento de los tiempos quirúrgicos (de 45,27 a 15,28 min en promedio) diferencia que al análisis estadístico resultó significativa, con relativamente pocas complicaciones $(14 \%)$ en relación a la técnica abierta en nuestras manos $(27 \%)$ y acorde con lo publicado en la literatura internacional $(7 \%$ a $19 \%$ de complicaciones en técnica percutánea), con un episodio de falsa vía que fue reconocido y solucionado oportunamente sin complicaciones y en que posiblemente influyó la poca experiencia del ejecutante (su segundo procedimiento de este tipo) y una tráquea anormalmente calcificada encontrada al momento de realizar el procedimiento abierto.

El uso del nasofibroscopio resultó imprescindible al inicio del proceso de aprendizaje, pasando luego a ser una medida de seguridad fundamental que siempre debe estar disponible para ser utilizado ante cualquier duda que se presente durante la realización del procedimiento, pero que será utilizado según el criterio del cirujano ya sea para revisar la posición del alambre de Seldinger o de la cánula ante la sospecha de una falsa vía.

El procedimiento posee una curva de aprendizaje relativamente rápida que permitió en nuestra experiencia hacerlo extensivo en un período de 4 años a prácticamente todos los pacientes que requieren de traqueostomía, lográndose una rápida 
adopción de la técnica por parte de los miembros del equipo, influyendo en su indicación más allá de las características morfológicas del cuello, la posibilidad de un adecuado reconocimiento de la vía aérea por palpación.

En lo que llevamos realizando el procedimiento, se nos han presentado una serie de inconvenientes menores que nos parece adecuado mencionar para ayudar a aquellos que incorporen la técnica a evitarlos o reconocerlos y solucionarlos precozmente:

- El inconveniente más frecuente es pinchar el tubo orotraqueal durante la punción de la vía aérea por retiro insuficiente de éste. Su suave movilización por el anestesista se transmite a nuestra aguja y confirma este hecho. Para su solución se debe retirar la aguja de punción con suavidad, revisando su permeabilidad antes de repetir la punción, pues ocasionalmente puede quedar obstruido el lumen por un trozo de material del tubo; luego realizar una nueva punción habiendo retirado el tubo orotraqueal a una posición más cercana a las cuerdas vocales y/o hacerla más caudal, afortunadamente en el último tiempo gracias a la adquisición por el Departamento de Anestesia de un videolaringoscopio, la posición del tubo puede ser monitorizada por el cirujano con ayuda del anestesista lo que logra un mejor posicionamiento del tubo y evita este problema.

- Cuando al introducir el alambre de Seldinger, su paso más allá de la bránula encuentra resistencia, debe plantearse la posible mal posición de ésta y repetir la punción y/o realizar una nasofibroscopía para revisar la posición de la guía.

- En caso de que una vez introducida la cánula de TQT, posicionada la endocánula y conectada al sistema de ventilación anestésico no se obtenga curva de capnografía, debemos verificar que el cuff de la cánula esté inflado y si persiste la duda del posicionamiento, revisar con fibroscopio a través de la cánula, para descartar una falsa vía.

- Se debe evitar el esparcimiento del lubricante del dilatador único más allá de la marca de introducción, pues ello puede hacer a éste muy resbaloso y difícil de manipular.
- Excepcionalmente se han presentado sangramientos menores peritraqueostoma especialmente en pacientes con coagulopatía 0 tratamiento anticoagulante, para evitarlos debemos reducir la movilización de la cánula fijándola con puntos a la piel y ocasionalmente hemos utilizado gasas con adrenalina en la zona sangrante o perlas de nitrato de plata para cauterizar algún vaso.

- La técnica percutánea no se debe utilizar en cicatrices previas de TQT pues la cicatriz impide la adecuada dilatación llevando a un elevado riesgo de falsa vía.

- Finalmente las recomendaciones de cuidado posoperatorio en esta técnica deben incluir la existencia al lado de la cama del enfermo, de una tijera que permita ante la sospecha de decanulación-obstrucción, la rápida sección de suturas, extracción de la cánula y reintubación del paciente.

\section{CONCLUSIONES}

En nuestra serie la traqueostomía percutánea, mostró ser un procedimiento seguro, que permitió un importante acortamiento del tiempo operatorio, que tiene una curva de aprendizaje relativamente rápida llegando a ser la técnica utilizada en más del $90 \%$ de los pacientes que requirieron el procedimiento una vez adquirida experiencia en ella. Por otro lado es nuestra especialidad la que reúne todas las competencias necesarias para su óptima ejecución y el adecuado manejo de sus eventuales complicaciones (procedimiento percutáneo, técnica abierta, uso de nasofibroscopio) por lo que deberíamos ser los encargados de su realización en todas las Unidades de Cuidados Intensivos del país.

\section{BIBLOGRAFÍA}

1. JaCkson C. Tracheostomy. Laryngoscope 1909; 19: 285-90.

2. Shelden CH, Pudenz RH, Fresshwater DB, Crue Bl. A new method for tracheostomy. J Neurosurg 1955; 12: 248-31.

3. Ciaglia P, Firsching R, Sinyec C. Elective percutaneous dilatational tracheostomy: a new 
simple bedside procedure; preliminary report. Chest 1985; 87: 715-9.

4. Johnson JL, Cheatham ML, Sagraves SG, Block EF, Nelson LD. Percutaneous dilatational tracheostomy: a comparison single- versus multiple-dilator techniques. Crit Care Med 2001; 29: 1251-4.

5. Byhahn $C$, Westphal $K$, Meininger D, Gürke $B$, KESSLER P, LISCHKE V. Single- Dilator percutaneous tracheostomy: a comparison PercuTwist and Ciaglia blue rhino techniques. Intensive Care Med 2002; 28: 1262-6.

6. Tonny Veenith, Sangeetha Ganeshamoorthy, Thomas Standley, Joseph Carter and Peter Young. Int Arch Med 2008; 1: 21.

7. K. Higgins laryngoscope 2007; 117: 447-54.

8. Eric R. Oliver. Laryngoscope 2007; 117: 15705.

9. Nasir Bhatti, Arzu Tatlipinar, Marek Mirski, Wayne
M. Koch, and David Goldenberg. OtolaryngologyHead and Neck Surgery 2007; 136: 938-41.

10. Carlos Romero P, Rodrigo Cornejo R, Mauricio Ruiz C, Ricardo Galvez A, Osvaldo llanos V, Eduardo Tobar A, Jorge Larrondo G, José Castro 0 . Traqueostomía percutánea con asistencia fibroscópica: Evaluacion retrospectiva de 100 casos consecutivos y revisión de la literatura. Rev Méd Chile 2008; 136: 1113-20.

11. Enrique Pérez B, Ricardo Yáñez M, Elizabeth Avilés C, Carlos Alarcón S, Osvaldo Weisse A, Maximiliano Curi T, Jorge Villalobos Z, Alejandro Palma M, Leopoldo Villarroel M. Traqueostomía percutánea en una Unidad de Cuidados Intensivos. Rev Chilena de Cirugía 2011; 63(4), 356-60.

12. Carlos Celedón L, Katherine Walker J, Alfredo Naser G, Paola Neumann M, Rodolfo Nazar S. Otorrinolaringol Cir Cabeza Cuello 2007; 67: 222-8.

Dirección: Ricardo Quitral C 